\title{
Automatic Detection of Modality with ITGETARUNS
}

\author{
Rodolfo Delmonte \\ Department of Language Studies \& Department of Computer Science \\ Ca' Foscari University - 30123, Venezia, Italy \\ delmont@unive.it
}

\begin{abstract}
In this paper we present a system for modality detection which is then used for Subjectivity and Factuality evaluation. The system has been tested lately on a task for Subjectivity and Irony detection in Italian tweets ${ }^{1}$, where the performance was $10^{\text {th }}$ and $4^{\text {th }}$, respectively, over 27 participants overall. We will focus our paper on an internal evaluation where we considered three national newspapers Il Corriere, Repubblica, Libero. This task was prompted by a project on the evaluation of press stylistic features in political discourse. The project used newspaper articles from the same sources over a period of three months, thus including latest political 2013 governmental crisis. We intended to produce a similar experiment and evaluate results in comparison with previous 2011 crisis. In this evaluation, we focused on Subjectivity, Polarity and Factuality which include Modality evaluation. Final graphs at the end of the paper will show results confirming our previous findings about differences in style, with $I l$ Corriere emerging as the most atypical.
\end{abstract}

\section{Introduction}

In this paper we present a system for modality detection which uses the output of a deep dependency parser of Italian. The system focuses on the semantics associated to the Verbal Complex in order to tell whether the event described and the participants associated to the event are related to a fact that took place in the world or not. Modality in our system can also refer to the degree of certainty a speaker associated to the current proposition or sentence. This is turned into a set of features which are then used for Subjectivity $($ see, $[11 ; 12 ; 16 ; 17 ; 18])$ and Factuality evaluation.

Modality in the Italian Verb Complex can be expressed by Modal Verbs and by fully inflected verbs, thus referrable by Mood and Tense morphological features. Classical subdivision of modality is into four main classes: POSSIBILITY, NECESSITY, OBLIGATION and PERMISSION. In addition to these classes Mood and Tense may express modality content when associated to Aspectual information. We tap this information directly from our lexicon of Italian, MIDUV. Additional information is gathered from special periphrastic constructions for PROGRESSIVE made of STARE PER, STARE Verb+gerund. Typical modality triggering Moods are CONDITIONAL and SUBJUNCTIVE; as to modal Tense, we consider all those related to the FUTURE or Irrealis mode. In addition we also assign modality markers to QUESTIONS and IMPERATIVES. Other typical modality markers are assigned to ADVERBIALS appropriately classified in our lexicon of Italian for modality. Modality is an attribute of the event expressed by a proposition that is crucial for the assessment of FACTUALITY. However, we also consider other attributes related to the assessment of SUBJECTIVITY of paramount importance for Modality detection. We assume that Modality is always related to an attitude by the speaker to express some degree of uncertainty in the statement he/she is formulating. In particular we are referring to specific classes of verbs like Mental Activity verbs, Presuppositional verbs, Opacity inducing or Intensional verbs: Hope, Want, Wish, Seem, Appear, Desire, Believe, Think etc. which are also coincident with the class of Attitudinal verbs. Modality related grammatical structures include Hypotheticals or Conditionals clauses triggered by the presence of a discourse marker (see $[8 ; 15])$.

The paper is organized as follows: in section 2, we briefly present the system; in section 3 we discuss and propose annotation examples from the database of sentences we evaluated; in section 4 we discuss previous work and the experiment we did with 6000 sentences. Then the evaluation and some conclusion.

\section{The System ITGETARUNS}

In this section we present a detailed description of the system for Italian that we used in this experiment. The system is derived from GETARUNS, a multilingual system for deep text understanding with limited domain dependent vocabulary and semantics, that works for English, German and Italian and has been developed in the past 20 years or so and documented in several publications and conference presentations $[3 ; 4 ; 5 ; 6 ; 7]$. The current version used for Italian has been made possible by the creation of the needed semantic resources, in particular a version of SentiWordNet[8] adapted to Italian and heavily corrected and modified. SentiWordNet derives from the English WordNet and the mapping of sentiment weights has been done automatically starting from the

$1 \quad$ http://www.di.unito.it/ tutreeb/sentipolc-evalita14/index.html 
linguistic content of WordNet glosses. However, this process has introduced a lot of noise in the final results, and many entries have a totally wrong opinion evaluation. So we started to correct and optimize the resource for our domain, i.e. newswire politically oriented text.

We modified the classification in order to characterize uniquely all those entries that have a "generic" or "commonplace" positive, or negative meaning associated to them in the specific domain. This was deemed the only possible solution to the problem of semantic ambiguity, which could only be solved by introducing a phase of Word Sense Disambiguation which was not part of the system. However this was not possible for all entries. So, we decided to erase all entries that had multiple concepts associated to the same lemma, and had conflicting sentiment values. We also created and added an ad hoc lexicon for the majority of concepts (some 3000) contained in the texts we analysed, in order to increase the coverage of the lexicon. This was done again with the same approach, i.e. labelling only those concepts which were uniquely intended as one or the other sentiment, restricting reference to the domain of political discourse.

The system has been lately documented by our participation in the EVALITA (Evaluation of NLP and Speech Tools for Italian) challenge ${ }^{2}$. It works in a usual NLP pipeline: the system tokenizes the raw text and then searches for Multiwords. The creation of multiwords is paramount to understanding specific domain-related meanings associated to sequences of words. This procedure is then extended to NER (Named Entity Recognition), which is performed on the basis of a big database of entities, lately released by JRC (Joint Research Centre) research centre. ${ }^{3} \mathrm{We}$ also use our own list of entities and multiwords some $100 \mathrm{~K}$ entries. Words that are not recognized by simple matching procedures in the big wordform dictionary (500K entries), are then passed to the morphological analyser. In case also this may fail, the guesser is activated, which will at first strip the word of its affixes. It will start by stripping possible prefixes and then analysing the remaining portion; then it will continue by stripping possible suffixes. If none of these succeeds, the word will be labelled by a backoff procedure, as foreign word if the final character is not a vowel; a noun otherwise. We then perform tagging and chunking. In order to proceed to the semantic level, each nominal expression is classified at first on the basis of the assigned tag: proper nouns are classified in the NER task. The remaining nominal expressions are classified using classes derived from ItalWordNet (Italian WordNet $)^{4}$. In addition to that, we have compiled specialized terminology databases for a number of specific domains including: medical, juridical, political, economic, and military. These lexica are used to add a specific class label to the general ones derived from ItalWordNet. And in case the word or multiword is not present there, to uniquely classify them. The output of this semantic classification phase is a vector of features associated to the word and lemma, together with sentence index and sentence position. Semantic mapping is then produced by a linguistically based dependency parser. In particular, we use a subcategorized lexicon of Italian verbs of some $17 \mathrm{~K}$ entries to choose between argument labels like SUBJ, OBJ2, OBL which are used for core arguments, and ADJ which is used for all adjuncts requires some additional information related to the type of governing verb. The first element for Modality annotation is the Verbal Complex(hence VC), which contains all linguistic items that may contribute to its semantic interpretation, including auxiliaries, modals, adverbials, negation, clitics. We then distinguish passive from active diathesis and we use the remaining information available in the feature vector to produce a full-fledged semantic classification at propositional level. Semantic mapping includes, beside diathesis:

- Change in the World; Subjectivity and Point of View; Speech Act; Factuality; Polarity.

At first we compute Mood and Tense from the VC which, as said before, may contain auxiliaries, modals, clitics, negation and possibly adverbials in between. From Mood_Tense we derive a label that is the compound tense and this is then used together with Aspectual lexical properties of the main verb to compute Change_in_the_World. This results in a subclassification of events into three subclasses: Static, Gradual, Culminating. From Change_in_the_World we compute (Point_of_)View, which can be either Internal (Extensional/Intensional) or External. Internal View then allows a labeling of the VC as Subjective for Subjectivity and otherwise, Objective (more details below). Eventually, we look for negation which can be produced by presence of a negative particle or be directly in the verb meaning as lexicalised negation. Negation, View and Semantic Class, together with presence of absence of Adverbial factual markers are then used to produce a Factuality labeling.

One important secondary effect that carries over from this local labeling, is a higher level propositional level ability to determine inferential links intervening between propositions. Whenever we detect possible dependencies between adjacent VCs we check to see whether the preceding verb belongs to the class of implicatives. We are here referring to verbs such as "refuse, reject, hamper, prevent, hinder, etc." on the one side, and "manage, oblige, cause, provoke, etc." on the other. In the first case, the implication is that the action described in the complement clause is not factual. In the second case, the opposite will apply.

http://www.evalita.it/

http://irmm.jrc.ec.europa.eu/

http://www.ilc.cnr.it/iwndb/iwndb_php/ 


\section{Modality Annotation: Some Examples}

Modality annotation hinges mainly upon lexical information, but has also the need of morphological and syntactic processing of the input sentence[1;2;10;13]. It is attached to a clause or a proposition, thus including both tensed and untensed VP structures. The main annotation items are three VIEW, FACTUALITY and CHANGE, and they are organized as shown here below:

VIEW: Function/Semantic_Role, Speech_Act, Semantic_Category, Diathesis, Verb_Predicate, Support_Verb

FACTUALITY: Verb_Predicate, Tense, Mood, Function/Semantic_Role, Support_Verb

CHANGE: Speech_Act, Polarity, Support_Verb, View, Mood, Tense, Aspect

In the examples below, will use the term Factivity and factive rather than Factuality and factual. Annotations may contain more than one Modality attribute, so MODAL1 may include additional features presented above: "forse"/maybe, will be annotated modall="probable"; "mica"/at_all as modal1=negated; deontic DOVERE/Must or Has_to, as modal1="deontic". All degree modifiers are also included under MODAL1 with a feature derived from [13] and taken from the following list: Intensifier, Maximizer, Booster, Approximator, Compromiser, Diminisher, Minimizer, Exclusivizer. They all contribute to Subjective interpretation. Notice that Subjective interpretation will also follow from the union of two components: View=internal and Factivity=nonfactive. Here below some Italian examples and the related classification: examples are shown after they have been tokenized and multiwords have been automatically created by the system.

<testo_frase id="repubblica_1911822">, “Ancora ricordo l'incontro che feci in Transatlantico con Giorgio_Amendola."</testo_frase $>/$ I still remember the meeting I had in "Transatlantico" with Giorgio_Amendola

\begin{tabular}{|l|l|c|l|l|l|l|}
\hline Lemma & View & Word & Modal & Change & Factivity & Moodtense \\
\hline ricordare & internal & ricordo & & gradual & factive & present \\
\hline fare & internal & feci & & culminated & factive & past_tense \\
\hline
\end{tabular}

Below an example of deontinc modality and one case of Modal1: a deontic double modality example. Notice that even though dependency from a nonfactive matrix clause induces nonfactivity in a dependent clause, when a relative clause appears, this is not inherited.

<testo_frase id="corriere_318263">,"Sui Trasporti, può essere comodo scaricare l'aumento delle tariffe sulla Regione, ma una città come Roma deve poter decidere per conto proprio."”</testo_frase>/ About Transportation, it could be convenient to charge the rise in tarifs on the Region, but a city like Rome has to be able to decide by herself.

\begin{tabular}{|l|l|l|l|l|l|l|l|}
\hline Lemma & View & Word & Modal & Modal1 & Change & Factivity & Moodtense \\
\hline essere & external & essere & potere & & culminated & nonfactive & perfect \\
\hline scaricare & external & scaricare & & & null & nonfactive & infinitive \\
\hline decidere & internal & decidere & potere & deontic & null & nonfactive & infinitive \\
\hline
\end{tabular}

\section{Previous Analysis and the Experiment}

We will focus now on the evaluation of newswire articles where we classified Subjectivity and Factuality which include Modality evaluation. Final graphs at the end of the paper show results and evaluation, confirming our previous findings in differences in style, with Il Corriere emerging as the most atypical. We decided to evaluate manually the data produced by our system and this was the topic of a Master thesis which was also checked personally by myself. The experimental setup required a smaller amount of data to be checked manually and a clear indication of choices to be made when annotating different types of modality. Instructions to the annotator were as follows:

- differentiate tensed propositions which can be computed as factives from untensed ones

- differentiate tensed propositions were modality was present in one or double feature and compute them as nonfactive

- differentiate gerundives and participials which must be computed as factives from infinitivals

- differentiate simple infinitivals from past or complex infinitivals which can be computed as factives

- differentiate propositions which are dependent from a nonfactive matrix clause from the rest

- check for lexically triggered subjectivity - semantically marked verb classes

The general quantitative data presented here below in fact show a similar situation to the previous 2011 evaluation. In fact, even though the database created was much smaller, only 6000 sentences compared to 20000 
of the previous experiment, we can clearly see that the number of nonfactive and subjective propositions in $I l$ Corriere is much higher in absolute numbers than the ones of the other two newpapers. It constitutes the $37 \%$ against the $29 \%$ of Libero and the $34 \%$ of Repubblica. Similar proportions can be found for Subjectivity, where Corriere has again $36 \%$ against $30 \%$ of Libero and $34 \%$ of Repubblica.

\begin{tabular}{|c|c|c|c|c|c|c|}
\hline Newspapers & Tot.Subject & Tot.Nonfact & Errs.Nonfact & Errs.Subject & No.Sents & No.Propos.Structs. \\
\hline Corriere & 1377 & 2504 & 236 & 196 & 1804 & 5514 \\
\hline Libero & 1142 & 1971 & 159 & 47 & 1965 & 4424 \\
\hline Repubblica & 1290 & 2264 & 152 & 36 & 2042 & 5048 \\
\hline TOTALS & 3809 & 6739 & 547 & 279 & 5811 & 14986 \\
\hline
\end{tabular}

Table 1. Quantitative overall data of the experiment for Subjectivity and Nonfactivity Evaluation

Results in the form of weighted data of the evaluation are shown in the graphs in Fig. 1 and 2. In Table 1. we also counted mistakes under Errs. since mistakes in tagging and in dependency parsing may affect the final outcome. Mistakes in automatic annotation of semantic features are strongly related to error propagation in the pipeline that constitutes the system. Additional errors are caused by problems in the semantic predicateargument structure building process where in some cases verbs have been wrongly collapsed into one single Verb Complex even though they constituted separated items. However, error percentages for nonfactivity is overall $8.2 \%$; while errors percentages for subjectivity is slightly lower, at $7.35 \%$. As can be noticed from Table 1. and graphs below, Corriere is by far the more difficult newspaper to analyse in terms of semantic features. The great majority of errors are present in Corriere which also has the highest number of propositions but the lowest number of sentences. This amounts to saying that sentences in Corriere are much longer and more complex to read. When compared to number of propositions we see a different distribution of data, with $I l$ Corriere having the highest number of nonfactive proposition but Libero having the highest number of Subjective propositions.

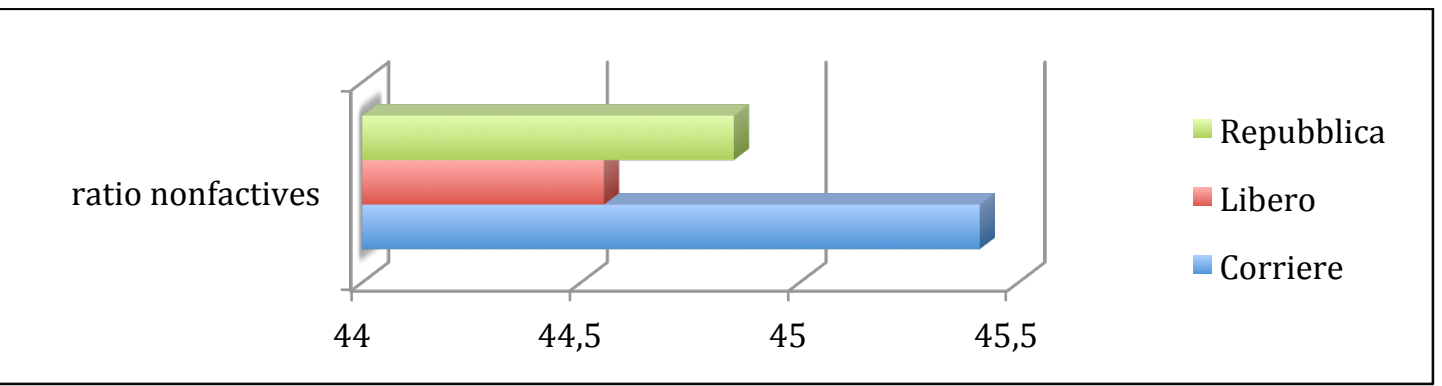

Fig. 1. Proportion of nonfactive propositions for the three newspapers

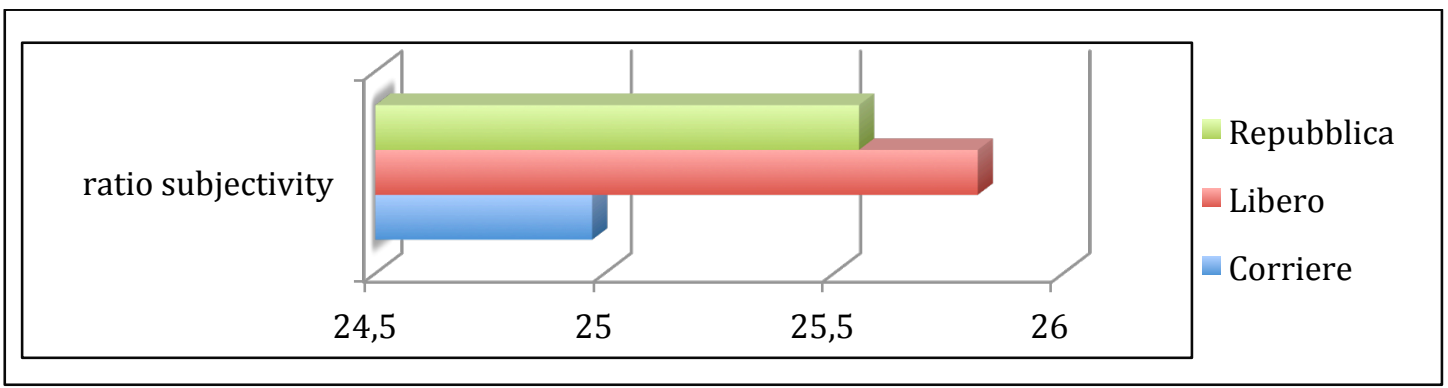

Fig. 2. Proportion of subjective propositions for the three newspapers

\section{Conclusion and Future Work}

We have presented work carried out to organize experiments intended to evaluate the ability of a system for deep dependency parsing of Italian to detect two semantic features, Factuality and Subjectivity, which are particularly sensitive to presence of modality at propositional level. We have presented the system and previous work done. We have shown in detail how the automatic annotation works and the types of different modality operators that can be represented by the system. Finally we have presented the experiment and the results obtained. In the future we intend to improve building of the Verbal Complex and the parser and tagger output. 


\section{REFERENCES}

1. Baker, K., Bloodgood, M., Dorr, B. J., Filardo, N. W., Levin, L., \& Piatko, C. (2014). A modality lexicon and its use in automatic tagging. arXiv preprint arXiv:1410.4868.

2. Bracewell, D., Hinote, D., \& Monahan, S. (2014, March). The Author Perspective Model for Classifying Deontic Modality in Events. In The Twenty-Seventh International Flairs Conference.

3. Delmonte R., 2013. Extracting Opinion and Factivity from Italian political discourse, in Bernadette Sharp, Michael Zock, (eds), Proceedings 10th International Workshop NLPCS, Natual Language Processing and Cognitive Science, 162-176, Marseille.

4. Delmonte R., Daniela Gifu, Rocco Tripodi, 2013. Opinion and Factivity Analysis of Italian political discourse, in Roberto Basili, Fabrizio Sebastiani, Giovanni Semeraro (eds.), Proc. 4th Italian Information Retrieval Workshop, IIR2013, Pisa. CEUR Workshop Proceedings (CEUR-WS.org), ISSN 1613-0073, http://ceur-ws.org, vol. 964, pp.88-99.

5. Delmonte R. and Vincenzo Pallotta, 2011. Opinion Mining and Sentiment Analysis Need Text Understanding, in Pallotta, Vincenzo; Soro, Alessandro; Vargiu, Eloisa (Eds.), "Advances in Distributed Agent-based Retrieval Tools: Studies in Computational Intelligence, Vol. 361, Springer, 81-96.

6. Delmonte R., 2007. Computational Linguistic Text Processing, Nova Science Publishers, New York.

7. Delmonte, R., 2004. Text Understanding with GETARUNS for Q/A and Summarization, Proc. ACL 2004 - 2nd Workshop on Text Meaning \& Interpretation, Barcelona, Columbia University, 97-104.

8. Doddington, G. R.; Mitchell, A.; Przybocki, M. A.; Ramshaw, L. A.; Strassel, S.; and Weischedel, R. M. 2004. The automatic content extraction (ace) program-tasks, data, and evaluation. In LREC.

9. Esuli A. and F. Sebastiani, EACL-06, 2006. Determining Term Subjectivity and Term Orientation for Opinion Mining, EACL-06, 2006.

10. Frawley, W (ed.). 2006. The expression of modality. Berlin: Mouton de Gruyter.

11. Kim S. and E. Hovy. Automatic Identification of Pro and Con Reasons in Online Reviews. COLING/ACL 2006.

12. Kobayashi N., R. Iida, K. Inui and Y. Matsumoto. Opinion Mining on the Web by Extracting Subject-AttributeValue Relations. In Proc. of AAAI-CAAW'06, 2006.

13. Nuyts, Jan. 2006. Modality: Overview and linguistic issues. in Frawley (ed.), 1-26.

14. Quirk R., Greenbaum S., Leech G., Svartvik J. (1985). A Comprehensive Grammar of the English Language. Longman: London.

15. Walker, C.; Strassel, S.; Medero, J.; and Maeda, K. 2006. Ace 2005 multilingual training corpus. Linguistic Data Consortium, Philadelphia.

16. Wilson T., J. Wiebe, and R. Hwa. Just how mad are you? Finding strong and weak opinion clauses. AAAI'04, 2004.

17. Wiebe J., and R. Mihalcea. Word Sense and Subjectivity. In ACL'06, 2006.

18. Wiebe J., and E. Riloff: Creating Subjective and Objective sentence classifiers from unannotated texts. CICLing, 2005. 